\title{
Time and Frequency Split Zak Transform for Finite Gabor Expansion
}

\author{
Soo-Chang Pei and Min-Hung Yeh \\ Department of Electrical Engineering \\ National Taiwan University \\ Taipei, Taiwan, R. O. C.
}

\begin{abstract}
The relationship between finite discrete Zak transfor$m$ and finite Gabor expansion are well discussed in this paper. In this paper, we present two DFT-based algorithms for computing Gabor coefficients. One is based upon the time-split Zak transform, the other is frequencysplit Zak transform. These two methods are time and frequency dual pairs. Furthermore, we extend the relationship between finite discrete Zak transform and Gabor expansion to the 2-D case and compute 2-D Gabor expansion coefficients through 2-D discrete Zak transform and 4-D DFT. Four methods can be applied in the 2D case. They are time-time-split, time-frequency-split, frequency-time-split and frequency-frequency-split.
\end{abstract}

\section{Introduction}

A time-frequency mapping, Zak transform, has been be used to calculate Gabor coefficients efficiently in critical sampling [1]. Recently, Zibulski and Zeevi have proposed a method which is based upon Zak transform and frame concept [4] to calculate the Gabor coefficients in oversampling case. The work of this paper is extending the theories proposed by Zibulski and Zeevi to discrete case and developing DFT-based algorithms for computing Gabor coefficients efficiently in oversampling scheme. One is based upon time-split Zak transform, the other is based upon frequency-split Zak transform. The timesplit algorithm is the same as that proposed in [7], but it is independently developed. The two-dimensional Gabor expansion has been widely used in image analysis and compression [2]. But the problem of computation burden is a more serious case. In this paper, we present four DFT-based algorithms for computing 2-D Gabor coeffcients to compute Gabor coefficients in oversampling case through Zak transform.

II. Review of Gabor expansion and finite discrete Zak transform

The discrete version. for the finite or periodic se- quences is defined as $[3]$ :

$$
\tilde{f}(i)=\sum_{m=0}^{M-1} \sum_{n=0}^{N-1} C_{m, n} \tilde{h}_{m, n}(i)
$$

where

$$
\begin{aligned}
C_{m, n} & =\sum_{i=0}^{L-1} \tilde{f}(i) \tilde{\gamma}_{m, n}^{*}(i) \\
\tilde{h}_{m, n}(i) & =\tilde{h}(i-m \Delta M) W_{L}^{n \Delta N i} \\
\tilde{\gamma}_{m, n}(i) & =\tilde{\gamma}(i-m \Delta M) W_{L}^{n \Delta N i} \\
W_{L}^{n \Delta N i} & =e^{j 2 \pi n \Delta N i / L}
\end{aligned}
$$

where $\tilde{f}(i), \bar{h}(i)$ and $\tilde{\gamma}(i)$ indicate the periodic extensions of $f(i), h(i)$ and $\gamma(i)$, respectively. $L$ is the number of sampling points in original signal. $M$ is the number of sampling points in time domain. $\triangle M$ is time sampling interval. $N$ is the number of sampling points in frequency domain. $\triangle N$ is frequency sampling interval. $M \triangle M=L$, $N \triangle N=L$. The condition $\triangle M \cdot \triangle N \leq L$ must be satisfied for a stable reconstruction. The critical sampling occurs when $\triangle M \cdot \triangle N=M \cdot N=L . \triangle M \triangle N<L($ or $M N>L)$ is oversampling case. Define $\alpha=\frac{M N}{L}=\frac{N}{\Delta M}=\frac{M}{\Delta N}=$ $\frac{L}{\Delta M \Delta N}=\frac{q}{p}$. where $\alpha$ is called the oversampling ratio, and $p, q$ are relative prime integers. The values, $\frac{\Delta M}{p}$ and $\frac{\Delta N}{p}$, are integers. The above defined finite discrete Gabor expansion is called $(M, N)$-point Gabor expansion in our further discussion.

The discrete Zak transform for discrete signal is defined as [1]:

$$
\hat{f}\left(\frac{a}{A}, \frac{b}{B}\right)=\sum_{r=-\infty}^{\infty} f\left(\frac{a}{A}+r\right) e^{-j 2 \pi \frac{-b}{b}}
$$

where $0 \leq b<B, 0 \leq a<A$. For convenience of further discussion, the index in definition of discrete Zak transform has been changed to integers in this paper. If the 
signal is $L=A \times B$ periodic or finite with length $L$, its definition becomes

$$
\hat{f}_{(A, B)}(a, b)=\sum_{r=0}^{B-1} f(a+A r) W_{L}^{-r b A}
$$

where $0 \leq b<B, 0 \leq a<A$ and $W_{L}=e^{\frac{j 2 \pi}{2}}$. We call this transform to be $(A, \bar{B})$-point finite discrete Zak transform (FZT) in this paper. The discrete signal $f(i)$ performed by $(A, B)$-FZT is denoted by $\hat{f}_{(A, B)}$ in the following discussions. The discrete signal $f(i)$ can be recovered from by inverse finite discrete Zak transform (IFZT).

$$
f(a+A r)=\frac{1}{B} \sum_{b=0}^{B-1} \hat{f}_{(A, B)}(a, b) e^{j 2 \pi \frac{r b}{B}}
$$

where $0 \leq a<A, 0 \leq r<B$.

III. Relationship between 1-D discrete Gabor expansion and 1-D FZT

In the critical sampling case, the discrete $(M, N)$ point Gabor expansion can be obtained through $(N, M)$ point 1-D FZT [1]. The numbers of time and frequency samples are interchanged for Gabor expansion and FZT. The FZT of analysis basis function is

$$
\hat{\gamma}_{(N, M)}(a, b)=\frac{1}{N \hat{h}_{(N, M)}^{*}(a, b)}
$$

In general case, the Gabor coefficients can be evaluated through the following equation:

$$
C_{m, n}=\frac{1}{L} \sum_{a=0}^{N-1} \sum_{b=0}^{M-1} \frac{\hat{f}_{(N, M)}(a, b)}{\hat{h}_{(N, M)}(a, b)} W_{L}^{-a n M+b m N}
$$

where $0 \leq m<M, 0 \leq n<N$. Equation (6) indicates the Gabor coefficients can be evaluated through a 2-D DFT from FZT of analysis basis and signal.

Applying the frame operator in [4] and [5], Zibulski and Zeevi have developed an algorithm for computing Gabor coefficients of continuous signal in oversampling case from continuous $\mathrm{Zak}$ transform domain.

$$
\gamma=S^{-1} h
$$

Now two methods for computing discrete Gabor analysis basis function and coefficients will be introduced for oversampling scheme. The discrete $(M, N)$-point Gabor expansion can be evaluated by two methods: $(N, \Delta N)$ and $(\triangle M, M)$-point FZT. The time-split method is to utilize $(N, \triangle N)$-point FZT. Thus the FZT of analysis basis function for the case $p=1$ is equal to:

$$
\hat{\gamma}_{(N, \Delta N)}(a, b)=\frac{q}{N} \frac{\hat{h}_{(N, \Delta N)}(a, b)}{\sum_{l=0}^{q-1}\left|\hat{h}_{(N, \Delta N)}(a-l \Delta M, b)\right|^{2}}
$$

where $0 \leq a<N, 0 \leq b<\Delta N$. We can calculate the IFZT for $\hat{\gamma}_{(N, \Delta N)}(a, b)$ to get $\gamma(i)$. The Gabor expansion coefficients in general case are:

$$
\begin{aligned}
C_{m, n}= & \frac{1}{\Delta N} \sum_{a=0}^{N-1} \sum_{b=0}^{\Delta N-1} \hat{f}_{(N, \Delta N)}(a, b) \hat{\gamma}_{(N, \Delta N)}^{*}(a-m \Delta M, b) W_{N}^{-n a} \\
& =\frac{1}{\Delta N} \sum_{a=0}^{N-1} \sum_{b=0}^{\Delta N / p-1}\left[\sum_{d=0}^{p-1} \hat{f}_{(N, \Delta N)}\left(a, b+d \frac{\Delta N}{p}\right)\right. \\
& \left.\hat{\gamma}_{(N, \Delta N)}^{*}\left(a-v \Delta M, b+d \frac{\triangle N}{p}\right)\right] \cdot W_{L / p}^{b u N-n a \Delta N / p}
\end{aligned}
$$

where $m=u \cdot q+v, 0 \leq m<M, 0 \leq n<N, 0 \leq u<\Delta N / p$, $0 \leq v<q$. The equation (9) indicates that the Gabor coefficients in time-split method can be obtained through $q$ amount of operations, which are $(N, \triangle N / p)$-point 2-D DFT. The $v$-th 2-D DFT is to compute the $(u q+v)$-th time slice of Gabor coefficients. $(0 \leq u<\triangle N / p, 0 \leq$ $v<q)$ Analysis algorithm of time-split method is liste $\bar{d}$ below:

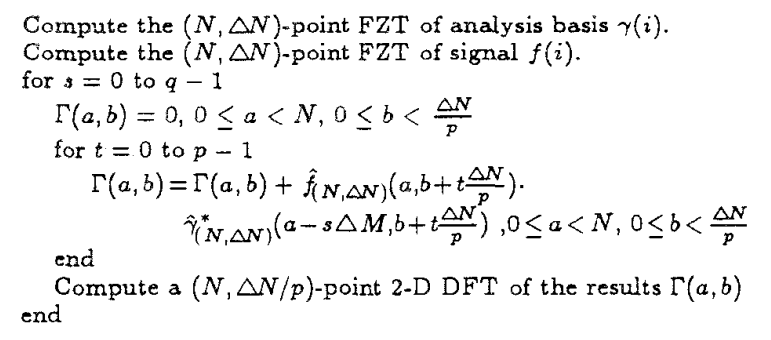

The reconstruction of function from its Gabor coefficients is given by

$$
\hat{f}_{(N, \Delta N)}(a, b)=\sum_{m=0}^{M-1 N-1} \sum_{n=0} C_{m, n} \hat{h}_{(N, \Delta N)}(a-m \Delta M, b) W_{N}^{a n}
$$

where $0 \leq a<N, 0 \leq b<\Delta N$

$$
\begin{aligned}
& \hat{f}_{(N, \Delta N)}\left(a, b+t \frac{\Delta N}{p}\right) \\
&=\sum_{s=0}^{q-1} \hat{h}_{(N, \Delta N)}\left(a-s \Delta M, b+t \frac{\Delta N}{p}\right) \\
& \sum_{n=0}^{N-1} \sum_{m=0}^{\frac{\Delta N}{p}-1} C_{m q+s, n} W_{L / p}^{a n \frac{\Delta N}{p}-b \Delta m N}
\end{aligned}
$$

where $0 \leq a<N, 0 \leq b<\frac{\Delta N}{p}, 0 \leq d<p$. Synthesis algorithm of time-split method is liseted below:

\footnotetext{
Compute the $(N, \triangle N)$-point FZT of synthesis basis $h(i)$.

for $s=0$ to $q-1$

Compute the 2-D DFT of $C_{m a t s, n}$

$F(a, b)=f f t 2\left(C_{m a+s, n}\right)_{m, n)->(a, b)} 0 \leq m<\frac{\Delta N}{p}, 0 \leq n<N$

$\Gamma(a, b)=0,0 \leq a<N, 0 \leq b<\frac{\Delta N}{p}$
} 


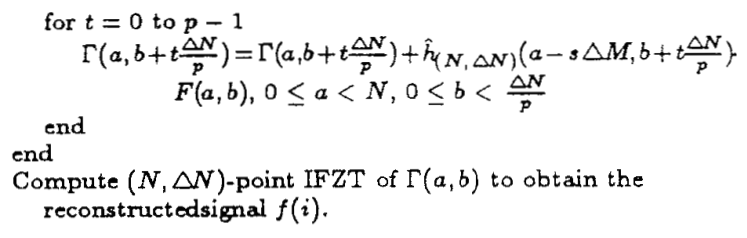

The frequency-split method is to utilize $(\triangle M, M)$-point FZT. The FZT of analysis basis function for $p=1$ in frequency-split method is:

$$
\hat{\gamma}_{(\Delta M, M)}(a, b)=\frac{q}{\Delta M} \frac{\hat{h}_{(\Delta M, M)}(a, b)}{\sum_{l=0}^{q-1}\left|\hat{h}_{(\Delta M, M)}(a, b+l \triangle N)\right|^{2}}
$$

where $0 \leq a<\triangle M, 0 \leq b<M$. The Gabor expansion coefficients in general case are:

$$
\begin{array}{r}
C_{m, n}=\frac{1}{M} \sum_{a=0}^{\Delta M-1} \sum_{b=0}^{M-1} \hat{f}_{(\Delta M, M}(a, b) \hat{\gamma}_{(\Delta M, M)}^{*}(a-m \Delta M, b-n \Delta N) W_{N}^{-n a} \\
=\frac{1}{M} \sum_{a=0}^{\Delta M / p} \sum_{b=0}^{M-1}\left[\sum_{d=0}^{p-1} \hat{f}_{(\Delta M, M)}\left(a+d \frac{\Delta M}{p}, b+v \Delta N\right)\right. \\
\left.\hat{\gamma}_{(\Delta M, M)}^{*}(a, b) W_{L / p}^{-a v \Delta N}\right] \cdot W_{L}^{b m \Delta M / p-a u M}
\end{array}
$$

where $0<m<M, 0<n<N, n=u \cdot q+v, 0<v<q$ and $0 \leq u<\Delta M / p$. The equation (12) indicates that the Gabor coefficients in frequency-split method can be calculated through $q$ amount of operations, which are $(\triangle M / p, M)$-point 2-D DFT. The $v$-th 2-D DFT is to compute the $(u \cdot q+v)$-th frequency slice of Gabor coefficients. $\left(0 \leq u<\frac{\Delta M}{p}, 0 \leq v<q\right)$ Analysis algorithm of frequency-split method is listed below:

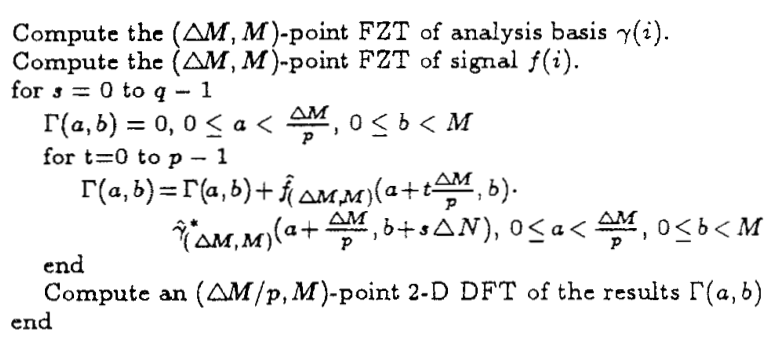

The reconstruction of signal $f(i)$ can be obtained from the Gabor coefficients.

$\hat{f}_{(\triangle M, M)}(a, b)=\sum_{m=0}^{M-1} \sum_{n=0}^{N-1} c_{m, n} \hat{h}_{(\triangle M, M)}(a-m \triangle M, b-n \triangle N) W_{N}^{a n}$

where $0 \leq a<\triangle M, 0 \leq b<M$

$$
\begin{aligned}
\hat{f}(\Delta M, M) & \left(d \frac{\Delta M}{p}+a, b\right) \\
= & \sum_{v=0}^{q-1}\left[\sum_{m=0}^{M-1} \sum_{u=0}^{\frac{\Delta M}{p}-1} C_{m, u q+v} W_{L / p}^{-(b-v \Delta N) m \Delta M / p+a u M}\right] \\
& \hat{h}_{(\Delta M, M)}\left(d \frac{\Delta M}{p}+a, b-v \Delta N\right) W_{L}^{a v \Delta N}
\end{aligned}
$$

where $0 \leq a<\frac{\Delta M}{p}, 0 \leq b<M$ and $0 \leq d<p$. Synthesis algorithm of frequency-split method is listed below

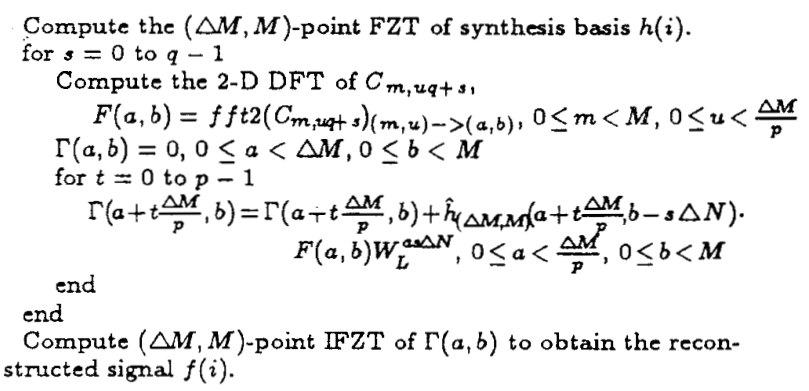

Example: $L=64, M=16, N=8, \Delta M=4, \Delta N=8$. The oversampling ratio in this example is 2 . The Gabor coefficients in time and frequency split methods be obtained through two 2-D DFT. In time-split method, $(8,8)$-point DFT and FZT are used. The first DFT compute $C_{0,0}, C_{0,1}, C_{0,2}, \cdots, C_{0,7}, C_{2,0}, C_{2,1}, \cdots, C_{14,7}$. The second DFT compute $C_{1,0}, C_{1,1}, C_{1,2}, \cdots, C_{1,7}, C_{3,0}$, $C_{3,1}, \cdots, C_{15,7}$. In frequency-split method $(4,16)$-point DFT and FZT are used. The first DFT compute $C_{0,0}$, $C_{1,0}, C_{2,0}, \cdots, C_{15,0}, C_{0,2}, C_{1,2}, \cdots, C_{15,6}$. The second DFT compute $C_{0,1}, C_{1,1}, C_{2,1}, \cdots, C_{15,1}, C_{0,3}, C_{1,3}, \cdots$, $C_{15,7}$.

In oversampling scheme, although the processes of time and frequency split methods are different, the results in these two methods are identical. Both methods are based upon the least square norm criterion.

\section{An extension to two-dimensional case for Zak} transform and Gabor expansion

The two methods that are proposed in the previous section for computing Gabor coefficients is suitable for finite signals. Image data is an example of two-dimensional finite signal. Now we will extend the above theories to the two-dimensional case.

The 2-D finite discrete Gabor expansion is defined as:

$$
\tilde{f}(x, y)=\sum_{\substack{m_{x}, n_{x} \\ m_{y}, n_{y}}} C_{m_{x}, n_{s} m_{y} n_{y}} h\left(x-m_{x} \Delta M_{x}, y-m_{y} \Delta M_{y}\right) W_{L}^{\left(n_{x} x+n_{y} y\right.}
$$

where $\tilde{f}(x, y)$ is periodic extension of $f(x, y), h(x, y)$ is the synthesis basis. $0 \leq m_{x}<M_{x}, 0 \leq m_{y}<M_{y}, 0 \leq$ $n_{x}<N_{x}$ and $0 \leq n_{y}<N_{y} . M_{x}$ and $M_{y}$ are the numbers of sampling points in time domain for $x$ direction and $y$ direction, respectively. $N_{x}$ and $N_{y}$ are the numbers of sampling points in frequency domain for $x$ direction and $y$ direction, respectively. $\Delta M_{x}$ are the time sampling intervals in $x$ direction and $y$ direction, respectively. $\Delta N_{x}$ and $\Delta N_{y}$ are frequency sampling intervals in $x$ direction $y$ direction, respectively. $M_{x} \cdot \Delta M_{x}=L_{x}, M_{y} \cdot \Delta M_{y}=$ $L_{y}, N_{x} \cdot \Delta N_{x}=L_{x}, N_{y} \cdot \Delta N_{y}=L_{y}$. The conditions, 
$\triangle M_{x} \Delta N_{x} \leq L_{x}$ and $\triangle M_{y} \Delta N_{y} \leq L_{y}$, must be satisfied for a stable reconstruction. The critical sampling case occurs when $M_{x} N_{x}=L_{x}$ and $M_{y} N_{y}=L_{y}$. Define $\alpha_{x}=$ $\frac{M_{x} N_{x}}{L_{x}}=\frac{g_{x}}{p_{x}}$ to be the oversampling ratio in $x$ direction, and $\alpha_{y}=\frac{M_{y} N_{y}}{L_{y}}=\frac{q_{y}}{p_{y}}$ to be the oversampling ratio in $y$ direction. 2-D FZT be extended from the definition of 1-D FZT directly. The 2-D FZT is defined as

$\hat{f}\left(a_{x}, a_{y}, b_{x}, b_{y}\right)=\sum_{k=0}^{B_{x}-1 B_{y}-1} \sum_{i=0} f\left(a_{x}+k A_{x}, a_{y}+l A_{y}\right) e^{-j 2 \pi\left(\frac{k b_{x}}{B_{x}}+\frac{l b_{y}}{k_{y}}\right)}$

where $0 \leq b_{x}<B_{x}, 0 \leq b_{y}<B_{y}, 0 \leq a_{x}<A_{x}$ and $0 \leq$ $a_{y}<A_{y}$. This 2-D FZT is called $\left(A_{x}, A_{y}, B_{x}, B_{y}\right)$-point 2-D FZT, and it is denoted by $\hat{f}_{\left(A_{x}, A_{y}, B_{x}, B_{y}\right)}$. For critical sampling case, $\left(M_{x}, M_{y}, N_{x}, N_{y}\right)$-point 2-D Gabor expansion can be obtained through $\left(N_{x}, N_{y}, M_{x}, M_{y}\right)$-point 2-D FZT [6].

In 1-D case, two methods based upon FZT for computing finite Gabor coefficients in oversampling scheme are presented. Thus, there exists four methods based on FZT for computing 2-D Gabor coefficients in oversampling scheme: $\left(N_{x}, N_{y}, \Delta N_{x}, \triangle N_{y}\right),\left(N_{x}, \triangle M_{y}, \triangle N_{x}\right.$, $\left.M_{y}\right),\left(\triangle M_{x}, N_{y}, M_{x}, \triangle N_{y}\right)$ and $\left(\triangle M_{x}, \triangle M_{y}, M_{x}, M_{y}\right)-$ point 2-D discrete Zak transform. They are named timetime-split, time-frequency-split, frequency-time-split and frequency-frequency-split, respectively. Their analysis bases and Gabor coefficients can be attained through the following equations:

time-time-split method :

The Gabor coefficients in this method can be obtained through $\left(N_{x}, N_{y}, \triangle N_{x}, \triangle N_{y}\right)$-point FZT and $\left(q_{x} \cdot q_{y}\right)$ operations, which are $\left(N_{x}, N_{y}, \triangle N_{x} / p_{x}\right.$, $\left.\triangle N_{y} / p_{y}\right)$-point 4-D DFT calculation.

time-frequency-split method :

The Gabor coefficients in this method can be obtained through $\left(N_{x}, \Delta M_{y}, \triangle N_{x}, M_{y}\right)$-point FZT and $\left(q_{x} \cdot q_{y}\right)$ operations, which are $\left(N_{x}, \triangle M_{y} / p_{y}\right.$, $\left.\triangle N_{x} / p_{x}, M_{y}\right)$-point 4-D DFT calculation.

frequency-time-split method :

The Gabor coefficients in this method can be obtained through $\left(\triangle M_{x}, N_{y}, M_{x}, \triangle N_{y}\right)$-point FZT and $\left(q_{x} \cdot q_{y}\right)$ operations, which can be $\left(\triangle M_{x} / p_{x}, N_{y}, M_{x}\right.$, $\left.\triangle N_{y} / p_{y}\right)$-point 4-D DFT calculations.

frequency-frequency-split method :

The Gabor coefficients in this method can be obtained through $\left(\triangle M_{x}, \triangle M_{y}, M_{x}, M_{y}\right)$-point FZT and $\left(q_{x} \cdot q_{y}\right)$ operations, which are $\left(\triangle M_{x} / p_{x}\right.$, $\left.\triangle M_{y} / p_{y}, M_{x}, M_{y}\right)$-point 4-D DFT calculations.

Similar to the 1-D case. the above four methods will result the same analysis basis function and Gabor coefficients.

\section{Conclusion}

According to the above discussion, several conclusions can be made: First, the $(M, N)$-point finite Gabor expansion can be obtained by using $(N, M)$-point FZT in critical sampling case. In oversampling case, $(M, N)$-point finite Gabor expansion can be obtained by two methods: One is calculated by $(N, \triangle N)$-point FZT, the other is obtained by $(\triangle M, M)$-point FZT. These two methods will result the same analysis bases and Gabor coefficients. If the oversampling ratio increases, the analysis bases will be more similar to the synthesis bases. The Gabor coefficients in the proposed two methods can be achieved through $q$ 2-D DFT operations.

2-D Gabor expansion has been widely used in image analysis and compression. We can extend the theories from 1-D case to the 2-D case and compute 2-D Gabor coefficients efficiently. Four DFT-based algorith$m$ for computing 2-D Gabor coefficients are attained to compute them efficiently.

\section{References}

[1] L. Auslander, I. C. Gertner, and R. Tolimieri, "The discrete Zak transform application to timefrequency analysis and synthesis of nonstationary signals," IEEE Trans. Signal Processing, vol. 39, pp. 825-835, April, 1991.

[2] M. Porat and Y. Zeevi,, "The generalized Gabor scheme of image representation in biological and machine vision," IEEE Trans. Patt. Anal. Mach. Intell., vol. 40, pp. 452-468, July, 1988.

[3] J. Wexler and S. Raz, "Discrete Gabor expansions," Signal Processing, vol. 21, pp. 207-220, 1990.

[4] M. Zibulski and Y. Y. Zeevi, "Oversampling in the Gabor scheme," IEEE Trans. Signal Processing, vol. 41, pp. 2679-2687, Aug., 1993.

[5] I. Daubechies, "The Wavelet transform, timefrequency localization and signal analysis," IEEE Trans. Information Theory, vol. 36, pp. 961-1005, Sep., 1990.

[6] Y. Zeevi and Gerner, "The finite Zak transform: An efficient tool for image representation, analysis," $J$. Visual Comm Image Representation vol. 3, pp. 1323, March 1992.

[7] M. Zibulski and Y. Zeevi, "Frame Analysis of the Discrete Gabor-Scheme" IEEE Trans. Signal Processing, vol. 42, pp. 942-945, April, 1994. 